\title{
Successful Management of Supraorbital Neuralgia Using Manual Therapy Technique: A Case Report
}

\author{
Nematollahi $\mathrm{M}^{*}$ \\ School of Rehabilitation Sciences, Department of Physiotherapy, Shiraz University of Medical Science, Shiraz, Iran
}

*Corresponding author: Nematollahi M, School of Rehabilitation Sciences, Department of Physiotherapy, Shiraz University of Medical Science, Shiraz, Iran, Tel: +987136271551, E-mail: neamatollahy@gmail.com

Citation: Nematollahi M (2018) Successful Management of Supraorbital Neuralgia Using Manual Therapy Technique: A Case Report. J Neurosci Neuropsyc 2: 102. doi: 10.18875/2577-7890.2.102

Article history: Received: 02 November 2017, Accepted: 07 March 2018, Published: 09 March 2018

\begin{abstract}
Background: Supraorbital neuralgia is a difficult to treat condition characterized by pain over the forehead area supplied by the supraorbital nerve, with concomitant tenderness over the supraorbital notch. Previous studies demonstrated that supraorbital neuralgia is not that amenable to pharmaceutical treatment. Non-drug interventions, such as surgery, are the treatment of choice in these patients, with accompanying side effects.
\end{abstract}

Case Presentation: In this case report, soft tissue therapy and friction massage was applied over the frontal area and supraorbital rim of a patient diagnosed with chronic and non-traumatic supraorbital and supratrochlear neuralgia with a resultant resolution of headaches. The pain was severe, disturbing the patient's sleep for several years. There was a lack of benefits from drug prescription, including sodium valproate.

Conclusion: Though the efficacy of physical therapy in the treatment of supraorbital neuralgia needs to be confirmed in well-designed trials, this case is presented as an example of how this non-invasive and cost-effective technique can harmlessly provide analgesia in a difficult to treat neuralgia. Promoting such interventions may prevent unnecessary surgery and excessive use of opioids and other painkillers in patient suffering from supraorbital neuralgia.

Keywords: Headache; Manual Therapy; Nerve entrapment; Supraorbital neuralgia

\section{Introduction}

Supraorbital and supratrochlear nerves are branches of frontal nerve, which emanate from the ophthalmic division of trigeminal nerve. These two nerves supply the skin over the forehead, upper eyelid and anterior scalp. The supraorbital nerve gives up a small branch in the supraorbital notch that supplies the mucosal membrane of the frontal sinus [1]. Hence, it is not uncommon for this neuralgia to be misdiagnosed as frontal sinusitis, as in the present case. Supraorbital and supratrochlear neuralgia is a rare disorder of adulthood. It is characterized by the International Classification of Headache Disorder-2 (ICHD-2) by the following criteria: pain in the region of supraorbital notch and forehead, tenderness over the supraorbital notch and absolute - but transitory - relief of symptoms upon supraorbital nerve blockage [2]. Previous studies demonstrated that nerve compression by overlying soft tissues is one of the main culprits of the supraorbital neuralgia. There is lack of physiotherapy intervention in the literature regarding this issue. Pharmaceutical management in supraorbital neuralgia is not as effective as in other headache disorders [3]. This leads to considering invasive alternatives such as nerve excision [4]. In the present case report, a patient with severe pain extending to the area supplied by the supraorbital and supratrochlear nerves was treated successfully with physical therapy intervention.

\section{Case description}

A 52-year-old man with a 4-year history of severe stabbing pain (VAS=10) in the right frontal region, diagnosed as frontal sinusitis, was referred to a physiotherapy clinic in order to receive magnetic therapy. The patient complained of severe pain in the areas supplied by the supraorbital and supratrochlear nerves upon falling asleep, irrespective of sleeping position. The pain awakened the patient 15-20 minutes after falling asleep, and lasted roughly half an hour. The pain did not return again during the rest of the night and used to occur once every night. It was the case during diurnal sleep. Mild autonomic accompaniment comprising lacrimation and rhinorrhea were reported on the involved side. Other migrainous features such as nausea, vomiting and photophobia were not 
found. No other trigger mechanisms were identified. The patient did not report any history of trauma to the head and neurological disorders. Active and passive cervical range of motion was pain-free and within normal limit. On palpation, there was a tender area over the exit of supraorbital nerves at the supraorbital rim on the right side. Likewise, skin over the eyebrow in the area innervated by the supraorbital and supratrochlear nerves demonstrated significant tenderness on palpation. Moreover, skin over the right side of the forehead was tough and less pliable as compared to the left side. There were neither trigger points nor sensory deficit over the area. There was not any tenderness over other areas of the face, head or cervical area. Physical activities such as walking did not use to trigger the patient's condition. He was on nortriptyline for control of his chronic depression. The patient was formerly diagnosed as having migraines and prescribed sodium valproate, with scant benefit.

He was initially treated with Chinese traditional medicine (acupuncture) with minimal and transient relief. He was not on any medications concomitant with physiotherapy sessions. Magnetic Resonance Imaging of the brain was unremarkable, indicating that there was no intracranial or brainstem pathological conditions, including tumor or demyelinating diseases. His laboratory blood tests were within normal limit. There were no frontal sinusitis signs and symptoms. These characteristics favor supraorbital neuralgia rather than frontal sinusitis. The patient was then referred to a neurologist in order to verify possibility of supraorbital neuralgia. A diagnostic block was given by the physician to the supraorbital and supratrochlear nerves, culminating in pain relief for several days. The patient's pain was seven out of ten based on VAS the night before the block applied. The day after the block, he reported zero to one on VAS scale. Hence, the patient was diagnosed as supraorbital neuralgia.

Since soft tissue tightness was found during assessment, the patient referred back to physiotherapy in order to release tight tissue in frontal area. This was due to the fact that in the first visit the patient stated that he recently found that placing a warm towel and rubbing the frontal area would ease the pain. Therefore, the patient was recommended to come back to physiotherapy in order to release soft tissue in involved area irrespective of diagnosis.

In order to differentiate the effects of physical therapy and injection block, the patient was asked to come to the clinic when headache attacks started again. The patient returned three weeks after nerve block with pain six to seven out of ten. During physiotherapy sessions, friction massage and gentle soft tissue therapy applied over the supraorbital notch, supraorbital rim and forehead skin for ten minutes, for 10 sessions. With decreasing pain and irritability, the intensity and depth of friction massage and soft tissue release increased accordingly. No other modalities were used. On the first session, the patient reported considerable pain relief and was not being awakened during sleep. After 10 sessions, his pain and concomitant autonomic features were completely relieved and patient was discharged.

On discharge, the skin toughness was no longer appreciable over the forehead and skin tenacity was similar to that of a normal side. Moreover, tenderness over the supraorbital notch, supraorbital rim and forehead region had almost completely disappeared. When the patient was seen for follow-up two and five months after discharge, the pain no longer persisted. Highlights of the patient's history are summarized in the timeline (Figure 1).Consent to publish has been obtained from the patient.
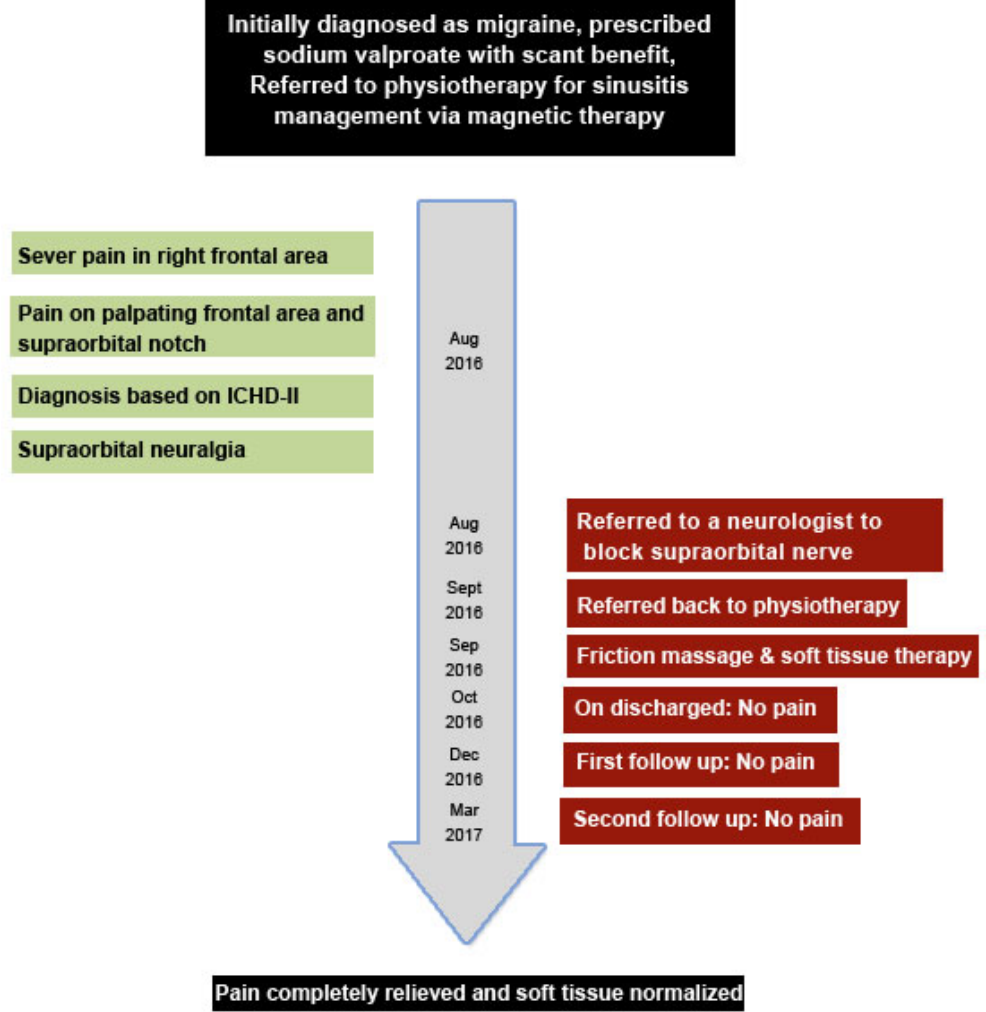

Figure 1: Timeline: Important information from the Patient History 


\section{Discussion}

Different hypotheses have been reported in literature as causes of supraorbital and supratrochlear neuralgia. Viral infections, demyelination, tumors and trauma are some that can be mentioned, with nerve compression being the most common causative factor, as in the present case. Since Magnetic Resonance Imaging was normal, intracranial causes were ruled out. Furthermore, palpable toughness of the skin over supraorbital notch and forehead skin on the right side, accentuated extracranial source of headache. This is in accordance with previous investigations, which reported unilateral protracted forehead in patients diagnosed with supraorbital neuralgia. They reported symptoms relief following liberation of the supraorbital nerve from surrounding tissue band via surgical procedure [3]. Other researchers also attributed the supraorbital neuralgia to the scar tissue and muscle entrapment, suggesting that soft tissue tightness over the forehead may be one of the main causes of supraorbital neuralgia [5-6]. Similarly, dramatic pain relief after Botox injections have been reported in patients with supraorbital neuralgia, suggesting that muscle stiffness may entrap supraorbital and supratrochlear nerves [7]. Although etiology of the supraorbital and supratrochlear neuralgia is almost unknown, entrapment of the nerve at its outlet may be a causative factor, signifying an extracranial headache, amenable to physical therapy intervention. The possible causes of soft tissue tightness over the forehead and supraorbital rim in supraorbital neuralgia are not clear. However, releasing this tightness may unload the embedded supraorbital and supratrochlear nerves.

Several approaches are common in treating supraorbital and supratrochlear neuralgia, depending on the causes and patient's condition. Injection of local anesthesia and surgical decompression are two prevailing approaches in many patients. To the best of the author's knowledge, this was the first time that a patient with supraorbital and supratrochlear neuralgia was treated with a manual technique. Since pain and other autonomic dysfunctions disappeared following above-mentioned intervention, supraorbital neuralgia in this case can be attributed to the soft tissue tightness in the supraorbital notch and frontal area. Hence, the present case can be considered as a secondary supraorbital neuralgia [2]. As compared to anesthetic injection, which is transient and focuses on alleviating symptoms, this approach may be considered a causative remedy dealing with the main cause of nerve compression, i.e. soft tissue stiffness. Previous studies showed that peripheral nerve stimulation is an effective alternative for treating supraorbital neuralgia. However, this is an invasive and possibly expensive approach [8].

In this case, pain was reported while sleeping, which is considered a rare characteristic of trigeminal neuralgia [9]. However, the present case is considered as peripheral nerve entrapment and sleep disturbance is not far from expectation in such circumstances [10].

Although autonomic features are less likely in supraorbital neuralgia, previous investigation reported that mild cranial autonomic activation may be seen in first division trigeminal neuralgia $[2,11]$. This case has differential diagnosis with hypnic headache and autonomic trigeminal cephalalgias. However, the above mentioned criteria are more consistent with that of supraorbital neuralgia. Furthermore, there are similarities between cluster headaches and supraorbital neuralgia. However, while the present case is considered a supraorbital neuralgia with an extracranial origin, the former is attributed more to the intracranial factors. Among possible interventions dealing with extracranial source of headache, there is a lack of physiotherapy approach, possibly due to lack of referral of such patients to physiotherapy clinics. This case report is an example of successful treatment of supraorbital and supratrochlear neuralgia using physical therapy intervention. This intervention would be effective in reducing medication intake and invasive surgery in patients suffering from the supraorbital and supratrochlear neuralgia with extracranial origin. Moreover, the technique presented here, may be considered as a confirming tool to differentiate intracranial and extracranial sources of headache. The efficacy of this technique needs to be verified in a well-designed trial.

\section{Conclusion}

Sound diagnosis of supraorbital and supratrochlear neuralgia and distinguishing its source of pain is of utmost importance in choosing the best approach of treatment. Physiotherapy may be a treatment of choice if soft tissue stiffness entraps the nerve extracranially.

\section{References}

1. Cuzalina AL, Holmes JD (2005) A simple and reliable landmark for identification of the supraorbital nerve in surgery of the forehead: an in vivo anatomical study. J Oral Maxillofac Surg 63: 25-7.

2. Olesen J, Steiner TJ (2004) The international classification of headache disorders, 2nd edn (ICDH-II). J Neurolo Neurosur Psychiatry 75: 808-11.

3. Sjaastad O, Stolt-Nielsen A, Pareja JA, Fredriksen TA, Vincent M (1999) Supraorbital neuralgia. On the clinical manifestations and a possible therapeutic approach. Headache 39: 204-12.

4. Ducic I, Larson EE (2008) Posttraumatic headache: surgical management of supraorbital neuralgia. Plast reconstr surg 121: 1943-8.

5. Andersen NB, Bovim G, Sjaastad O (2001) The frontotemporal peripheral nerves.Topographic variations of the supraorbital, supratrochlear and auriculotemporal nerves and their possible clinical significance. Surg Radiol Anat 23: 97-104

6. Simons DG, Travell JG, Simons LS (1999) Myofascial pain and dysfunction: upper half of body ( $2^{\text {nd }}$ Edn) Lippincott Williams \& Wilkins, USA.

7. Trescot AM (2000) Headache management in an interventional pain practice. Pain Physician 3: 197-200.

8. Amin S, Buvanendran A, Park KS, Kroin JS, Moric M (2008) Peripheral nerve stimulator for the treatment of supraorbital neuralgia: a retrospective case series. Cephalalgia 28: 355-9. 
9. Scrivani SJ, Keith DA, Mathews ES, Kaban LB (1999) Percutaneous stereotactic differential radiofrequency thermal rhizotomy for the treatment of trigeminal neuralgia. J Oral Maxillofac Surg 57: 104-11.

10. Lehtinen I, Kirjavainen T, Hurme M, Lauerma H, Martikainen K, et al. (1996) Sleep-related disorders in carpal tunnel syndrome. Acta Neurol Scand 93: 360-5.

11. Goadsby PJ, Matharu MS, Boes CJ (2001) SUNCT syndrome or trigeminal neuralgia with lacrimation. Cephalalgia 21: 82-3. 